\title{
Padrão de identidade e qualidade do tomate (Lycopersicon esculentum Mill.) de mesa
}

\author{
Identity and quality standards of tomatoes (Lycopersicon esculentum Mill.) for fresh consumption
}

\author{
Sila Mary Rodrigues Ferreira ${ }^{1}$ Renato João Sossela de Freitas ${ }^{2}$ Elisa Noemberg Lazzari ${ }^{3}$
}

\section{- REVISÃO BILBIOGRÁFICA -}

\section{RESUMO}

O valor comercial do tomate, Lycopersicon esculentum Mill., é definido pelas características de identidade e qualidade presentes no fruto que podem ser avaliadas pela classificação do produto. A presente revisão de literatura teve como objetivo descrever o padrão de identidade $e$ qualidade do tomate de mesa, através da classificação. $O$ tomate de mesa é classificado em grupos, subgrupos, classes e tipos. De acordo com o formato do fruto, o tomate é classificado em dois grupos: oblongo, quando o diâmetro longitudinal é maior que o transversal e redondo, quando o diâmetro longitudinal é menor ou igual ao transversal. Em função do estádio de maturação, o tomate é classificado em cinco subgrupos: verde maduro, pintado, rosado, vermelho $e$ vermelho maduro. A classe é definida em função do maior diâmetro transversal do fruto em relação ao grupo a que pertença. O tomate oblongo é classificado em três classes: grande, médio e pequeno. O tomate redondo é classificado em quatro classes: gigante, grande, médio e pequeno. De acordo com os índices de ocorrência de defeitos graves $e$ leves presentes nos frutos, o tomate é classificado também em tipo: extra, especial ou selecionado e comercial. São considerados defeitos graves: podridão, passado, queimado, dano por geada, podridão apical, e defeitos leves: dano, mancha, ocado, deformado e imaturo. A amostra de tomate que ultrapasse os índices de defeitos previstos pela legislação do Ministério da Agricultura é classificada como fora do padrão.

Palavras-chave: classificação do tomate de mesa; Lycopersicon esculentum Mill., padrão de identidade e qualidade.

\section{ABSTRACT}

The commercial value of tomatoes (Lycopersicon esculentum Mill.), is defined by the fruit's identity and quality characteristics, which can be evaluated by product classification. The present literature review had as objective to describe the identity and quality standards of tomatoes for fresh consumption, through the classification. The tomatoes for fresh consumption are classified in groups, subgroups, classes and types. According to the fruit's shape, tomatoes are classified in two groups: oblong, when the longitudinal diameter is greater than the transversal diameter; and round, when the longitudinal diameter is smaller or equal to the transversal diameter. According to the fruit's maturity, it is classified in five subgroups: mature green, breaker, pink, red and mature red. The class is determined by the greatest transversal diameter inside the same group. Oblong tomatoes are classified in three classes: big, medium and small. Round tomatoes are classified in four classes: giant, big, medium and small. According to the occurrence of serious damage and small defects on the fruit, it is classified in types, which can be extra, special or selected and commercial. The following are considered serious damage: decay, extreme maturity, burns, frost bites and rottenness at blossom end. Stains, hollowness, deformities, immaturity and little damages are considered small defects. The sample is classified as not meeting standards if it exceeds the requirements stated by the Department of Agriculture.

Key words: tomato classification, Lycopersicon esculentum Mill., identity and quality standards.

\section{INTRODUÇÃO}

Do ponto de vista da ciência dos alimentos, a qualidade é composta pelas características que diferenciam unidades individuais de um produto, sendo significante a determinação do grau de aceitabilidade pelo comprador (CHITARRRA \& CHITARRA, 1990).

O conceito de qualidade do tomate se refere àqueles atributos que o consumidor consciente ou inconscientemente estima que o produto deve possuir.

${ }^{1}$ Nutricionista, Professora do Departamento de Nutrição da Universidade Federal do Paraná (UFPR), Doutoranda do Programa de Pós-graduação em Tecnologia de Alimentos, UFPR. Av. Lothário Meissner 3400, Campus III, Jardim Botânico, 80210-170, Curitiba, PR. E- mail: sila@milenio.com.br. Autor para correspondência.

${ }^{2}$ Engenheiro Químico, Doutor em Química pela UFPR, Professor do Programa de Pós-graduação em Tecnologia de Alimentos, UFPR.

${ }^{3}$ Acadêmica do Curso de Nutrição da UFPR e Bolsista PIBIC/UFPR. 
Porém, é necessário ampliar esse conceito de qualidade e aplicá-lo não só ao consumidor, mas também a todos que participam da cadeia produtiva, isto é, desde o cultivo até o consumo. Aos produtores, compete colher frutos de alto rendimento, resistentes às enfermidades, de bom aspecto e com poucos defeitos. Aos distribuidores, manter as características sensoriais do produto e propiciar eficiente armazenamento enquanto os consumidores determinam sua qualidade através da aparência, consistência, diâmetro transversal, inexistências de deformidades e outros atributos sensoriais (CASQUET, 1998).

Para o produto ser comercializado com qualidade, é desejável que seja classificado antes de ser submetido à venda. Em razão do exposto, a presente revisão de literatura teve como objetivo descrever o padrão de identidade e qualidade do tomate (Licopersicon esculentum Mill.) de mesa, através da classificação.

\section{PADRÃO DE IDENTIDADE E QUALIDADE}

Nos últimos anos, graças às novas tecnologias, aumentou o número de cultivares plantadas em escala comercial no centro-sul que gerou necessidade de adotar padrão para comercialização. Até meados de 70, as normas para padronização e classificação de tomate destinado ao mercado que eram realizadas por cooperativas, passaram a ser adotadas pelos mercados do centro-sul e tinham como base o comprimento, diâmetro dos frutos, coloração, brilho e outros parâmetros (FILGUEIRA, 1982). A partir dessa época, foi estabelecida uma norma oficial para padronização e classificação de tomate que, ao longo dos anos, já foi submetida a alterações.

Pela legislação vigente (BRASIL, 1995) e pela proposta no Anexo XVII da Portaria SARC $\mathrm{n}^{\circ}$ 085/02 do MAPA (BRASIL, 2002), o tomate é classificado em grupos, subgrupos, classes ou calibres, tipos ou grau de seleção.

\section{Classificação em grupos}

De acordo com o formato do fruto, o tomate é classificado em dois grupos: oblongo, quando o diâmetro longitudinal é maior que o transversal e redondo, quando o diâmetro longitudinal é menor ou igual ao transversal (BRASIL, 1995; BRASIL, 2002). Na norma da Comissão Econômica Européia (ECE, 2000) e no Codex alimentarius proposto (FAO, 2002), o tomate é classificado em quatro grupos: redondo, achatado com sulcos, oblongo ou alongado e tomate cereja, incluindo no último o tomate cocktail. Forma bem definida, seja redonda, globosa, globosa planada ou ovalada dependendo da cultivar, juntamente com outros atributos, é um bom índice de qualidade do produto (CASQUET, 1998). A forma do tomate está relacionada ao grupo a que pertence a cultivar. Cultivares do grupo Santa Cruz apresentam frutos de formato oblongo ou alongado e predominantemente bilocular, tolerando-se todavia a presença de três lóculos e do grupo salada ou caqui que possuem formato redondo, globoso ou achatado são tipicamente pluriloculares (FILGUEIRA, 1982; CAMARGO, 1992). As cultivares de tomate cereja, híbridos amarelos, laranja e cocktail, que também são da forma arredondada, apresentam frutos biloculares (SAKATA, 1998; EMBRAPA, 1993; SAKAMA, 2001).

\section{Definição da classe ou calibre}

Os frutos são avaliados pelo tamanho que, por sua vez, é medido através da circunferência ou diâmetro transversal (AMARAL JÚNIOR et al., 1997; FONTES et al., 2000), largura (AMARAL JÚNIOR et al., 1997), peso e volume (CHITARRA \& CHITARRA, 1990; FERREIRA et al., 2000). Produtos com características de tamanho e peso padronizados são mais fáceis de ser manuseados em grandes quantidades, pois apresentam menores perdas, produção mais rápida e melhor qualidade (CHITARRA \& CHITARRA, 1990). Quando a classificação é efetuada à mão, não apresenta dificuldades. Entretanto, quando é realizada mecanicamente por calibradores de orifícios circulares (SILVA, 2001), um número razoável de tomates de menor calibre caem em lotes de outros calibres. Neste caso, é indispensável proceder a eliminação manual dos frutos fora de calibre e a sua inclusão nos lotes correspondentes (GAYET et al., 1995). A classe ou calibres do tomate de mesa na legislação brasileira (BRASIL, 1995; BRASIL, 2002) é definida em função do diâmetro transversal do fruto, em $\mathrm{mm}$, de acordo com o grupo a que pertença. Nos Estados Unidos (USDA, 2002), essa classificação independe do grupo. O tomate oblongo é classificado em três classes: grande, médio e pequeno, conforme pode ser visualizado na tabela 1 .

Tabela 1 - Classes do tomate em função do diâmetro transversal

\begin{tabular}{lcc}
\hline $\begin{array}{l}\text { Classes } \\
\text { calibres }\end{array}$ & ou & Diâmetro transversal do fruto $(\mathrm{mm})$ \\
& Oblongo & Redondo \\
\hline $\begin{array}{l}\text { Gigante } \\
\text { Grande }\end{array}$ & - & Maior que 100 \\
Médio & Maior que 60 & Maior que 80 até 100 \\
Pequeno & Maior que 50 até 60 & Maior que 65 até 80 \\
Maior que 40 até 50 & Maior que 50 até 65 \\
\hline
\end{tabular}

FONTE: BRASIL, 1995; BRASIL, 2002. 
O tomate redondo, com exceção do Lycopersicon esculentum, variedades cerasiforme, cereja (BRASIL, 1995; BRASIL, 2002), híbrido amarelo, alaranjado e cocktail, é classificado em quatro classes: gigante, grande, médio e pequeno (Tabela 1). Em ambos grupos, a diferença entre o diâmetro do maior e do menor fruto, em cada embalagem, não pode exceder a $15 \mathrm{~mm}$. É permitida a mistura de tomates pertencentes a classes diferentes, desde que a somatória das unidades não supere $10 \%$ e que pertençam à classe imediatamente superior e/ou inferior. O número de embalagens que exceder essa tolerância não pode ser superior a $20 \%$ das unidades encontradas no estoque (BRASIL, 1995; BRASIL, 2002).

FERREIRA, et al. (2000) classificaram amostras de tomates de procedência da região metropolitana de Curitiba, comercializadas no CEASA, do grupo redondo e consumidas no Hospital de Clínicas (HC), do grupo oblongo, respectivamente, e verificaram que as amostras apresentaram as seguintes concentrações de frutos nas classes: $3,33 \%$ e $29,66 \%$ pequeno; $79,0 \%$ e $57,0 \%$ médio; $17,33 \%$ e $13,33 \%$ grande. A amostra CEASA apresentou $0,33 \%$ de frutos da classe gigante.

Os atributos largura e altura dos frutos do tomateiro variam em função da cultivar. FLORI (1993), estudando essas características em tomate salada, observou que a variedade Tropicana apresentou maior largura e o híbrido $\mathrm{F}$ maior altura em relação aos oito híbridos experimentaís e duas testemunhas analisadas. $\mathrm{O}$ mesmo autor encontrou menos de $6 \%$ de frutos com diâmetro maior que $105 \mathrm{~mm}$ e 39 \% de diâmetro de 90-105mm.

O tamanho do fruto está relacionado entre outros fatores à quantidade de água utilizada na rega que determinará a maior ou menor concentração de componentes solúveis (CASQUET, 1998). Alto índice pluviométrico desencadeia uma produção de tomates de grande tamanho, porém com menor conteúdo de nutrientes e de sabor menos acentuado (CASQUET, 1998; SILVA \& GIORDANO, 2000).

O peso é outro atributo importante sob o ponto de vista comercial, apesar de estar relacionado à cultivar não é considerado pela legislação vigente. Tomate oblongo do grupo Santa Cruz apresenta peso médio de $60 \mathrm{~g}$ (PADOVANI, 1987) a $250 \mathrm{~g}$ (FILGUEIRA, 1982; CAMARGO, 1992; SAKATA, 1998; FERREIRA et al., 2000; SECRETARIA DE AGRICULTURA, IRRIGAÇÃO..., 2002), dependendo da cultivar e da região do plantio. Tomate redondo representado pelo grupo salada ou caqui apresenta peso médio de $140 \mathrm{~g}$ (CAMARGO, 1992) a 250g (FILGUEIRA, 1982; PADOVANI, 1987; CAMARGO, 1992; SAKATA, 1998;
FERREIRA et al., 2000; SECRETARIA DE AGRICULTURA, IRRIGAÇÃO..., 2002). Tomates cereja pesam em média de 15 a $25 \mathrm{~g}$, frutos do híbrido cocktail pesam $50 \mathrm{~g}$, enquanto que os híbridos amarelo e alaranjado produzem tomates pesando em média $75 \mathrm{~g}$ (SAKAMA, 2001).

Para FLORI (1993), frutos do grupo salada, com diâmetros de 60 a $75 \mathrm{~mm}$ e baixo peso, considerados pequenos, têm pouco valor comercial. Abaixo de 60mm, os frutos são miúdos e de pouco ou nenhum valor comercial. Frutos com diâmetro maior que $75 \mathrm{~mm}$ estão relacionados com a rentabilidade do produtor, pois frutos maiores, que representam o grupo salada, são preferidos pelo consumidor (MELO et al., 1988). Para o mercado exigente de tomate salada, são recomendados frutos extras, pois produtos médios e pequenos são descartados na comercialização (FLORI, 1993).

\section{Classificação em subgrupos}

Outro fator de qualidade do tomate é o estado fisiológico, que está relacionado com o estádio de maturação do fruto, pois é ele que define o momento da colheita. A cor sugere as mudanças de sabor, textura e aroma, decorrentes do processo de maturação (ZAMBON, 1984; SILVA \& GIORDANO, 2000). A modificação da coloração do tomate é devida à clorofila e aos carotenóides. A cor verde dos frutos imaturos é atribuída à clorofila. Ao máximo tamanho segue imediata mudança de cor, início da maturação, refletindo a degradação da clorofila, que permanece em pequena quantidade nos tecidos do fruto (MEDINA \& MEDINA, 1981; ZAMBON, 1984; ZAMBRANO et al., 1995). Os principais componentes dos carotenóides em tomate são o caroteno (amarelo) e o licopeno (vermelho), cuja síntese e decomposição são acentuadas na fase de transição entre a maturação e senescência do fruto (ZAMBON, 1984; ZAMBRANO et al., 1995). Em tomates, há intensa degradação de clorofila durante o amadurecimento, com síntese gradual de licopeno (CHITARRA \& CHITARRA, 1990). A cor vermelha dos frutos é considerada como sendo o acúmulo de licopeno. O estádio verde maduro (início de mudança de cor) é considerado o primeiro sintoma visual para o índice de maturação (ZAMBON, 1984).

A mudança de cor do tomate é considerada como índice de colheita (ZAMBRANO et al., 1995). Desde que o fruto tenha completado seu desenvolvimento fisiológico - esteja de vez -, poderá ser colhido, mesmo que se apresente com a coloração verde clara. $\mathrm{O}$ fruto fisiologicamente desenvolvido, verde maduro, ideal para a colheita, é identificado pela 
sua estrutura interna. As sementes devem estar completamente desenvolvidas e são cortadas pela lâmina ao se realizar um corte transversal do fruto. A placenta deve exibir um material gelatinoso em, pelo menos, um lóculo enquanto, nos demais, está em formação. O ponto de colheita determina maior ou menor resistência do fruto ao manuseio, sua capacidade de completar a maturação, sua aparência e qualidade (EMBRAPA, 1993; GAYET, et al., 1995; CASQUET, 1998).

A escolha do estádio de maturação depende das preferências regionais (FILGUEIRA, 1982; GAYET, et al., 1995), dos locais de comercialização (SECRETARIADEAGRICULTURAE IRRIGAÇÃO..., 2002) e emprego culinário (PAZINATO \& GALHARDO, 1997). Para mercados próximos, os tomates podem ser colhidos no estádio rosado ou vermelho maduro, enquanto para mercados distantes podem ser colhidos no estádio de maturação fisiológica verde maduro e completar sua maturação fora da planta (EMBRAPA, 1993; GAYET, et al., 1995; CASQUET, 1998), pois sendo o tomate um fruto climatérico pode desenvolver cor, aroma e sabor característicos nessa condição. A cor é o atributo de qualidade que serve de parâmetro para o consumidor. Dessa forma, a escolha, no ato da compra, recai sempre nos produtos mais coloridos. Há mercados, como de Goiânia, que preferem tomate verde maduro, já no Rio de Janeiro preferem frutos vermelhos ou avermelhados, bem firmes, enquanto em São Paulo, dão preferência a tomates na fase intermediária (FILGUEIRA, 1982; GAYET, et al., 1995).

De acordo com a Portaria no 553/95 do MAARA (BRASIL, 1995) e Anexo XVII da Portaria SARC no ${ }^{\circ}$ 085/02 do MAPA (BRASIL, 2002), o estádio de maturação, classifica o tomate de coloração vermelha, em cinco subgrupos: verde maduro, pintado, rosado, vermelho e vermelho maduro. Verde maduro: quando se evidencia o início do amarelecimento na região apical do fruto; pintado: quando as cores amarelo, rosa ou vermelho encontram-se entre $10 \%$ a $30 \%$ da superfície do fruto; rosado: quando $30 \%$ a $60 \%$ do fruto encontra-se vermelho; vermelho: quando o fruto apresenta entre $60 \%$ e $90 \%$ da sua superfície vermelha; e vermelho maduro: quando mais de $90 \%$ da superfície do fruto encontra-se vermelha. É permitido a mistura de até três colorações consecutivas em uma embalagem e no lote, o número de embalagem com a mistura não deve exceder a $20 \%$.

Na norma da Comissão Econômica Européia (ECE, 2000), não foram definidos os estádios de maturação do tomate. Porém, no Codex alimentarius proposto (FAO, 2002) e USDA (2002), respectivamente, o tomate é classificado em sete e seis grupos. No primeiro, estão sendo propostos os subgrupos: verde, verde maduro, levemente pintado, pintado (rajado), rosado, vermelho e vermelho maduro. No último, os subgrupos definidos são: verde, levemente pintado, pintado, rosado, vermelho claro e vermelho.

Tal como a legislação vigente (BRASIL, 1995) e a proposta no Anexo XVII da Portaria SARC no 085/02 do MAPA (BRASIL, 2002), a norma da Comissão Econômica Européia (ECE, 2000), anteprojeto do Codex alimentarius (FAO, 2002) e USDA (2002) não contemplam na definição do subgrupo, os frutos de coloração amarela e alaranjada.

A avaliação da cor é realizada na maioria dos produtores e/ou cooperativas por processo visual. Porém, há uma tendência para processo mecanizado através de câmaras cujas células fotoelétricas classificam cada tomate de acordo com quatro tamanhos e quatro cores diferentes, inclusive os totalmente verdes (SILVA, 2001).

Na investigação de FERREIRA et al. (2000), foi verificado que as amostras se encontram em estádios de maturação diferentes para a comercialização. A amostra denominada de CEASA, em frutos pertencentes ao grupo redondo, apresentou $15,0 \%$ de tomate verde maduro, $27,33 \%$ de pintados, $9,66 \%$ de rosado, $26,0 \%$ de vermelho e $22,0 \%$ de vermelho maduro. Já a amostra denominada de HC, pertencente ao grupo oblongo, não apresentou fruto verde, $3,33 \%$ de pintado, $18,33 \%$ de rosado, $63,0 \%$ de vermelho e $15,33 \%$ de vermelho maduro. Esses valores permitiram classificar as amostras no subgrupo pintado (CEASA) e vermelho (HC). A diferença do estádio de maturação entre as amostras, pode ser atribuída ao emprego culinário (PAZINATO \& GALHARDO, 1997) a que se destina a amostra $\mathrm{HC}$, já que se trata de frutos para ser prontamente utilizados no sistema de alimentação coletiva, enquanto a amostra CEASA pode ser levada em outros locais de comercialização (SECRETARIADE AGRICULTURAE IRRIGAÇÃO..., 2002) e ser revendida no varejo, necessitando de frutos menos maduros.

\section{Classificação em tipo}

A presença de defeitos ou danos reduz o potencial de comercialização, embora, em alguns casos, não haja redução do valor nutritivo e da qualidade comestível do produto. Os defeitos encontrados no tomate podem ser de origem fisiológica, entomológica, patológica e mecânica. Defeitos fisiológicos ocorrem devido a anomalias hereditárias ou podem ser atribuídos a condições externas desfavoráveis durante a fase de crescimento e maturação. Defeitos 
entomológicos são causados pela ação de insetos que provoquem danos diretos ou indiretos. Defeitos patológicos são atribuídos à ação de bactérias, fungos, leveduras ou vírus, que reduzem a qualidade do produto causando lesão, descoloração e podridão. Defeitos mecânicos são de natureza física em decorrência de manuseio inadequado provocando amassamento, corte ou machucadura (CHITARRA \& CHITARRA, 1990; CASTRO et al., 2001).

O tomate de mesa é classificado em tipo ou grau de seleção ou categoria em função dos defeitos graves e leves presentes nos frutos, conforme determina a Portaria no 553/95 do MAARA (BRASIL, 1995) e Anexo XVII da Portaria SARC no $085 / 02$ do MAPA (BRASIL, 2002). É classificado em tipo extra, categoria I ou especial ou selecionado e categoria II ou comercial segundo pode ser visto na tabela 2 .

São considerados defeitos graves: podridão, passado, queimado, dano por geada e/ou granizo e/ou baixas temperaturas e podridão apical. Os defeitos leves são: dano, mancha, ocado, deformado e imaturo (BRASIL, 1995; BRASIL, 2002). Nos Estados Unidos, segundo o USDA (2002), os defeitos são classificados, em relação ao grau de comprometimento da área atingida, em três níveis: dano, dano sério e dano muito sério. No Codex alimentarius proposto (FAO, 2002) e norma da Comissão Econômica Européia (ECE, 2000), os defeitos definem as classes em razão da análise qualitativa dos danos nos frutos.

FERREIRA et al. (2000) classificaram em tipo as amostras CEASA e HC e encontraram valores de $11,33 \%$ e $6,66 \%$ para o defeito podridão; $5,66 \%$ e $15,66 \%$ para passado; $54,33 \%$ e $30,0 \%$ para queimados; nenhum e $2,33 \%$ para danos por geado (frio); $2,33 \%$ e $4,33 \%$ de podridão apical; totalizando $73,66 \%$ e $58,68 \%$ defeitos graves e $130,65 \%$ (frutos que apresentaram mais de um defeito) e $64,65 \%$ defeitos leves, respectivamente. Esses valores quando confrontados com os limites da legislação vigente (BRASIL, 1995) classificaram as amostras como fora do padrão.
A qualidade dos frutos na fase pós-colheita depende dos recursos tecnológicos disponíveis na cadeia de comercialização. A seleção da tecnologia está relacionada ao tipo e destino do produto, devendo ser considerada a tecnologia apropriada para as condições locais e treinamento de pessoal. Procedimentos simples e baratos podem ser empregados de maneira que algumas práticas, como o manuseio cuidadoso na colheita, proteção contra a exposição do produto ao sol, colheita em período mais frio, ventilação adequada no container, embalagem e transporte adequado possam evitar injúrias (BRASIL, 1986; BRASIL, 1991; CASTRO et al., 2001). A adoção voluntária das normas de classificação é o caminho que leva transparência nas operações comerciais, permitindo a implantação de sistema confiável de informação de mercado, que possibilitará a modernização da comercialização (NÚCLEODENEGÓCIO, 1999). Porémé de fundamental importância que a legislação nacional e mundial de padrão de identidade e qualidade do tomate de mesa, acompanhe o desenvolvimento tecnológico de modo a contemplar as cultivares de diferentes formas, tamanhos e cores, conferindo condições plenas para o produto competir vantajosamente no mercado globalizado.

\section{CONCLUSÃO}

O valor comercial do tomate, Lycopersicon esculentum Mill., de mesa é definido pelas características de identidade e qualidade presente no fruto. O tomate é classificado em grupos, subgrupos, classes e tipos. Pelo formato, é classificado em dois grupos: oblongo, quando o diâmetro longitudinal é maior que o transversal e redondo quando o diâmetro longitudinal é menor ou igual ao transversal. Pelo estádio de maturação, o tomate é classificado em cinco subgrupos: verde maduro, pintado, rosado, vermelho e vermelho maduro. A classe é definida em função do diâmetro transversal do fruto em relação ao grupo a que pertença. $\mathrm{O}$ tomate oblongo é classificado em três classes: grande, médio e pequeno. $\mathrm{O}$ tomate redondo

Tabela 2 - Classificação do tomate em tipos

\begin{tabular}{|c|c|c|c|c|c|c|c|}
\hline \multicolumn{6}{|l|}{ Defeitos graves } & \multicolumn{2}{|c|}{ Total de defeitos $(\%)$} \\
\hline Tipos & Podridão & Passado & Queimado & Dano por geada & Podridão apical & Graves & Leves \\
\hline Extra & 0 & 1 & 1 & 1 & 1 & 2 & 5 \\
\hline Categoria I ou Especial & 1 & 3 & 2 & 2 & 1 & 4 & 10 \\
\hline $\begin{array}{l}\text { Categoria } \quad \text { II } \quad \text { ou } \\
\text { Comercial }\end{array}$ & 2 & 5 & 3 & 4 & 2 & 7 & 15 \\
\hline
\end{tabular}

FONTE: BRASIL, 1995; BRASIL, 2002

Ciência Rural, v. 34, n. 1, jan-fev, 2004. 
é classificado em quatro classes: gigante, grande, médio e pequeno. De acordo com a presença de defeitos graves e leves nos frutos, o tomate é classificado também em tipo: extra, especial ou selecionado e comercial.

Para permitir a certificação do produto em um sistema de qualidade, é necessário que as normas, nacional e mundial, do tomate de mesa se adaptem à realidade agrícola com vistas as cultivares de diferentes formatos, tamanhos e cores.

\section{REFERÊNCIAS BIBLIOGRÁFICAS}

AMARAL JÚNIOR, A.T. et al. Melhoramento do tomateiro: II. Procedimento de Gardner e Eberhart na análise heterótica de caracteísticas morfológicas e da qualidade dos frutos. Bragantia, Campinas, v.56, n.1, p.33-46, 1997.

BRASIL. Ministério da Agricultura do Abastecimento e da Reforma Agrária. Portaria no 553 de 30 de agosto de 1995. Dispõe sobre a Norma de Identidade, Qualidade, Acondicionamento e Embalagem do Tomate in natura, para fins de comercialização e Revoga as especificações de Identidade, Qualidade, Acondicionamento e Embalagem do Tomate, estabelecidas pela Portaria no. 76, de 25 de fevereiro de 1975. Diário Oficial da República Federativa do Brasil, Brasília, set, 1995.

BRASIL. Ministério da Agricultura do Abastecimento e da Reforma Agrária. Portaria no 127 de 04 de outubro de 1991. Aprova norma de embalagens para acondicionamento, manuseio, transporte, armazenamento e comercialização de produtos hortícolas destinado ao mercado atacadista. Diário Oficial da República Federativa do Brasil, Brasília, out, 1991.

BRASIL. Ministério da Agricultura. Portaria no 412 de 07 de outubro de 1986. Dispõe sobre o acondicionamento e embalagem, o uso de caixa de papelão ondulado para tomate destinado ao consumo in natura. Diário Oficial da República Federativa do Brasil do Brasil, Brasília, out, 1986.

BRASIL. Ministério da Agricultura, Pecuária e Abastecimento. Portaria SARC no 085 de 06 de março de 2002. Propõe o Regulamento técnico de identidade e qualidade para classificação do tomate. Diário Oficial da República Federativa do Brasil, Brasília, mar, 2002. (Consulta pública).

CAMARGO, L.S. As hortaliças e seu cultivo. 3.ed. Campinas : Fundação Cargill, 1992. 253p. (Série Técnica número 6).

CASQUET, E. Principios de economía agraria. Zaragoza: Acribia, 1998. 368p.

CASTRO, L.R.; CORTEZ, L.A.B.; JORGE, J.T. Influência da embalagem no desenvolvimento de injúrias mecânicas em tomates. Ciênc Tecnol Aliment, Campinas, v.21, n.1, p.2633, jan./abr, 2001.
CHITARra, M.I.; CHITARRA, A.B. Pós-colheita de frutos e hortaliças: fisiologia e manuseio. Lavras : ESAL/FAEPE, 1990. 320p.

ECONOMIC COMMISSION FOR EUROPE. UN/ECE standard ffv-36. Concerning the marketing and commercial quality control or tomatoes moving in international trade and to UN/ECE member countries. Committee for trade, industry and enterprise development. Genebra, 2000. p.27-30. (Report or the fitfy-sixth session, nov).

EMBRAPA. A cultura do tomateiro (para a mesa). Brasília: Embrapa - SPI, 1993. 92p.

FERREIRA, S.M.R. et al. Avaliação da qualidade do tomate. In: SIMPÓSIO SUL-BRASILEIRO DE NUTRIÇÃO, 1., 2000, Florianópolis. Anais: História, Ciência e Arte. Florianópolis : Pró Reitoria de Cultura e Extensão - UFSC, 2000. p.437- 441.

FILGUEIRA, F.A.R. Manual de olericultura: cultura e comercialização de hortaliças. 2.ed. São Paulo : Agronômica Ceres, 1982. V.2, 358p.

FLORI, J.E. Obtenção e avaliação de híbridos $\mathbf{F}$ de tomate (Licopersicon esculentum Mill) no grupo multilocular. 1993. 144f. Dissertação (Mestrado em Agronomia, área de concentração Fitotecnia) - Escola Superior de Agricultura de Lavras.

FONTES, P.C.R.; SAMPAIO, R.A.; FINGER, F.L. Fru it size, mineral composition and quality or trickle-irrigated tomatoes as affected by potassium rates. Pesq Agropec Bras, Brasília, v.35, n.1, p.21-25, jan, 2000.

FOOD AND AGRICULTURE ORGANIZATION OF THE UNITED NATIONS. Codex alimentarius commission. Proposed draft codex standard for tomatoes. Joint FAO/ OMS food standards programme. Disponível em: < $\underline{\text { http:// }}$ www.codexalimentarius.net/c10/ff02-01e.htm > Acesso em: 24 abr, 2002. 3p.

GAYET, J.P. et al. Tomate para exportação: procedimentos de colheita e pós-colheita. Ministério da Agricultura, do Abastecimento e da Reforma Agrária, Secretaria do Desenvolvimento Rural, Programa de Apoio à Produção e Exportação de Frutas, Hortaliças, Flores e Plantas Ornamentais. Brasília: EMBRAPA-SPI, 1995. 34p. (Série Publicações Técnicas FRUPEX: 13).

MEDINA, P.V.L.; MEDINA, R.M.T. Descrição bioquímica e fisiológica da maturação dos frutos do tomateiro. Revista Ceres, v.155, n.28, p.1-7, 1981.

MELO, P.C.T.; MIRANDA, J.E.C.; COSTA, C.P. Possibilidade e limitações do uso de híbridos $\mathrm{F}_{1}$ de tomate. Horticultura Brasileira, Brasília, v.6, n.2, p.4-7, nov, 1988.

NÚCLEO DE AGRONEGÓCIOS - CLASSIFICAÇÃO. Classificação do tomate. São Paulo : Programa de horti \& fruti padrão. Disponível em: <http://www.cati.sp.gov.br/ cotacao/classificacao/tomate.htm > Acesso em: 12 nov, 1999.

PADOVANI, M.I. Tomate. São Paulo : Ícone, 1987. p.2343. 
PAZINATO, B.C.; GAlHARDO, R.C. Processamento artesanal do tomate. $2^{\mathrm{a} i m p .}$ Campinas : Coordenadoria de Assistência Técnica Integral, 1997. 30p.

SAKAMA. Sementes sakama. Fornecendo produtos de qualidade: catálogo. São Paulo, [2001?]. p.5.

SAKATA. Sementes agroflora: catálogo 97/98. São Paulo, [1998?]. p.9-10.

SECRETARIA DE AGRICULTURA, IRRIGAÇÃO E REFORMA AGRÁRIA. EMBRAPA. Cultura-tomate. BAHIA Disponível em: <http://www.bahia.ba.gov.br/seagri/tomate.htm $>$ Acesso em: 8 mai, 2002.

SILVA, G. Seleção apurada. Revista Globo Rural, Jaguaré, v.16, n.191 p.59-61, set, 2001

SILVA, J.B.C.; GIORDANO, L.B. Tomate para processamento industrial. Brasília : Embrapa Comunica- ção para Transferência de Tecnologia - Embrapa Hortaliças, 2000. 168p.

UNITED STATES DEPARTAMENT OF AGRICULTURE. Fruits and vegetable division. United States standards for grades of fresh tomatoes. Reprinted - january 1997. Disponível em: < http:// www.ams.usda.gov/standerds.tomatfrh.pdf $>$ Acesso em: 29 abr, 2002

ZAMBON, F.R.A. Comparação dos processos de maturação de tomate (Lycopersicon esculetum, Mill.), Rada, Mutantes Nor e Rin e seus Hibridos F. 1984. $45 \mathrm{f}$ Dissertação (Mestrado em Fitotecnia) - Universidade Federal de Viçosa.

ZAMBRANO, J; MOYEJA, J.; PACHECO, L. Efecto del estado de madurez en la composición y calidad de frutos de tomate. Agronomia Tropical, Venezuela, v.46, n.1, p.61$72,1995$. 